\title{
A MATHEMATICAL FORMULATION FOR DIAMAGNETISM IN TETRAHEDRALLY BONDED SEMICONDUCTORS
}

\author{
M.A. GRADO-CAFFARO AND M. GRADO-CAFFARO \\ Julio Palacios 11, 28029 Madrid, Spain
}

(Received February 3, 1996; in final form March 29, 1996)

An expression for the magnetic susceptibility tensor corresponding to diamagnetic contribution in tetrahedrally bonded semiconductors is derived; this formulation refers to the crystalline state. In addition, a comparison with the amorphous case is outlined.

\section{INTRODUCTION}

A mathematical formulation for the magnetic susceptibility of a typical tetrahedrally bonded amorphous semiconductor has recently been performed [1]. Since an amorphous solid is isotropic, the magnetic susceptibility tensor may be expressed as $\overline{\bar{\chi}}=\chi \overline{\overline{1}}$. Therefore, we have $\chi_{\mathrm{xx}}=\chi_{\mathrm{yy}}=\chi_{\mathrm{zz}}$ and since $\overline{\bar{\chi}}=\overline{\bar{\chi}}^{(\mathrm{p})}+$ $\overline{\bar{\chi}}^{(\mathrm{d})}$, where $(\mathrm{p})$ denotes paramagnetic and (d) diamagnetic, we can write $\chi_{x x}^{(d)}=$ $\chi_{y y}^{(d)}=\chi_{z z}^{(d)}$. In the following, we will expose a formulation for the diamagnetic term of the susceptibility of a typical tetrahedral semiconductor in the crystalline state; since this state implies anisotropy, we have $\chi_{x x} \neq \chi_{y y} \neq \chi_{z z}$ so that $\chi_{x x}^{(d)} \neq$ $\chi_{y y}^{(d)} \neq \chi_{z z}^{(d)}$. The formulation in question is based on linear response theory and the Wannier model. On the other hand, it is well-known that in the amorphous state, disorder gives rise to a reduced paramagnetic contribution so that a net enhancement of diamagnetism takes place; this does not occur in the crystalline state.

\section{THEORY}

Assume a system with an eigenvalue spectrum formed by two bands so that [1]:

$\hat{\mathrm{H}} \mid \psi_{\mathrm{nn}}>=\mathrm{E}_{\mathrm{nn}} \cdot \psi_{\mathrm{nn}}>$

where Hdenotes hamiltonian, $\mathrm{n}$ is the band index ( $\mathrm{n}=0$ or 1$)$, and $\mathrm{n}^{\prime}$ denotes the states in the bands; eq. (1) is of course the time-independent Schrödinger equation. On the other hand, we assume that the ground state of the system is such that all the states in the $\mathbf{n}=0$ band are filled. Now we state:

$\overline{\bar{\chi}} \equiv\left(\begin{array}{ccc}\chi_{\mathrm{xx}} & 0 & 0 \\ 0 & \chi_{\mathrm{yy}} & 0 \\ 0 & 0 & \chi_{\mathrm{zz}}\end{array}\right)$ 
so that:

$\overline{\bar{\chi}}^{(\mathrm{d})} \equiv\left(\begin{array}{ccc}\chi_{\mathrm{xx}}^{(\mathrm{d})} & 0 & 0 \\ 0 & \chi_{\mathrm{yy}}^{(\mathrm{d})} & 0 \\ 0 & 0 & \chi_{\mathrm{zz}}^{(\mathrm{d})}\end{array}\right)$

by taking into account that the situation in which $\chi_{x y}^{(p)}+\chi_{x y}^{(d)}=0, \chi_{y z}^{(p)}+\chi_{y z}^{(d)}=0$ and $\chi_{x z}^{(p)}+\chi_{x z}^{(d)}=0$ is meaningless. We establish:

$\chi_{\mathrm{xx}}^{(\mathrm{d})}=-\alpha \sum_{\mathrm{i}}<\psi_{\mathrm{oi}}\left|\mathrm{y}^{2}+\mathrm{z}^{2}\right| \psi_{\mathrm{oi}}>$

$\chi_{\mathrm{yy}}^{(\mathrm{d})}=-\beta \sum_{\mathrm{i}}<\psi_{\mathrm{oi}}\left|\mathrm{x}^{2}+\mathrm{z}^{2}\right| \psi_{\mathrm{oi}}>$

$\chi_{\mathrm{zz}}^{(\mathrm{d})}=-\gamma \sum_{\mathrm{i}}<\psi_{\mathrm{oi}}\left|\mathrm{x}^{2}+\mathrm{y}^{2}\right| \psi_{\mathrm{oi}}>$

where $\alpha, \beta$ and $\gamma$ are real constants $(\alpha>0, \beta>0, \gamma>0)$. Note that the wave-function $\psi_{\mathrm{oi}}(\mathrm{x}, \mathrm{y}, \mathrm{z})$ fulfills eq. (1) so that $\hat{\mathrm{H}}\left|\psi_{\mathrm{oi}}>=\mathrm{E}_{\mathrm{oi}}\right| \psi_{\mathrm{oi}}>$. From (4), (5), and (6), it is deduced that

$\chi_{x x}^{(d)}=-\alpha \iiint_{D}\left(y^{2}+z^{2}\right)\left[\sum_{i}\left|\psi_{o i}(x, y, z)\right|^{2}\right] d x d y d z$

$\chi_{y y}^{(d)}=-\beta \iiint_{D}\left(x^{2}+z^{2}\right)\left[\sum_{i}\left|\psi_{o i}(x, y, z)\right|^{2}\right] d x d y d z$

$\chi_{z z}^{(d)}=-\gamma \iiint_{D}\left(x^{2}+y^{2}\right)\left[\sum_{i}\left|\psi_{o i}(x, y, z)\right|^{2}\right] d x d y d z$

where $\mathrm{D}$ is the spatial domain corresponding to the semiconductor sample considered. Eqs. (7), (8) and (9) constitutes a set of explicit formulae that becomes useful for numerical calculations.

\section{DISCUSSION}

In certain cases [1], for disordered states we have $\chi_{x x}^{(d)}=\chi_{y y}^{(d)}=0, \chi_{z z}^{(d)} \neq 0$; however, for the crystalline counterpart we have the preceding expressions. These formulae have been established from linear response theory and a Wannier representation. Our formulation is related to theoretical work on electron-spin resonance and associated problems developed in refs. [2] [3] [4]; this work refers to amorphous semiconductors. It is well-known that the tensor of electron-spin resonance is the $g$ 
tensor; this tensor is related to singly occupied dangling bond states. These states can be considered as deep levels in the gap of amorphous silicon because of three-fold coordinated $\mathrm{Si}$ atoms [4] [5].

\section{REFERENCES}

1. M. A. Grado-Caffaro and M. Grado-Caffaro. Act. Pass. Electronic Comp. 17, 253-255 (1994).

2. M. Stutzmann and D. K. Biegelsen. Phys. Rev. B 40, 9834 (1989).

3. S. Yamasaki, H. Okushi, A. Matsuda K. Tanaka, and J. Isoya. Phys. Rev. Lett. 65, 756 (1990).

4. M. A. Grado-Caffaro and M. Grado-Caffaro (unpublished).

5. M. H. Brodsky and R. S. Title. Phys. Rev. Lett. 23, 581 (1969). 

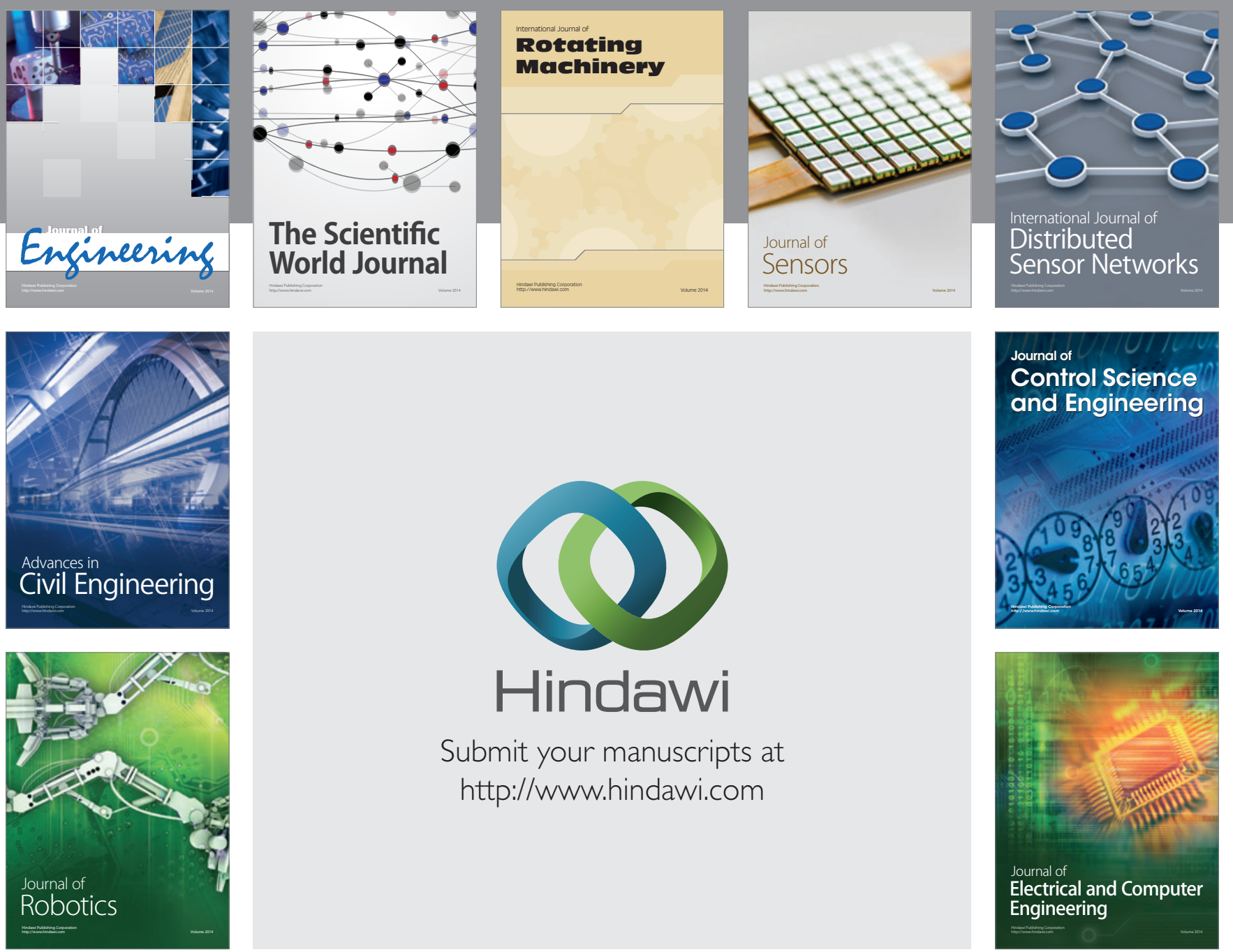

Submit your manuscripts at

http://www.hindawi.com
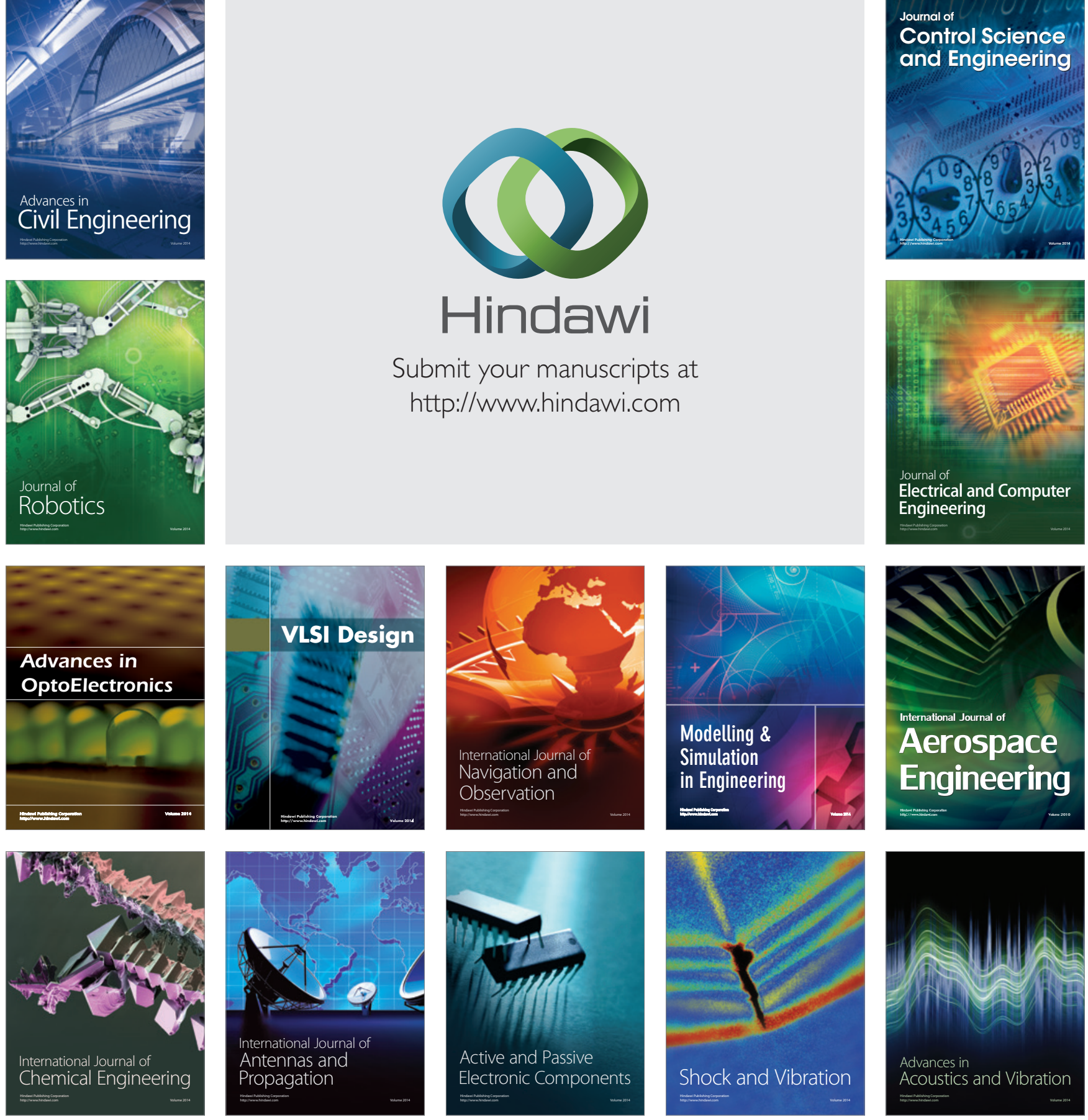